\title{
The Fusion Protein BnBI-1GFP is Localized to the Endoplasmic Reticulum (ER) and Allows Visualization of ER Reorganization Following Treatment with Salicylic Acid.
}

\section{Nathalie Bolduc* and Louise F. Brisson* \\ * Department of Biochemistry and Microbiology, Life and Health Sciences Research Building, Laval University, Québec, Canada, G1K 7P4.}

Programmed cell death (PCD) is a vital phenomenon for multicellular organisms because of its involvement in removing unnecessary or harmful cells during normal development or under pathological conditions [1]. In plants, PCD is involved in xylogenesis, leaf and flower senescence as well as during the establishment of the hypersensitive response [2]. To date, some regulators of plant PCD have been identified, but very few have been found to be homologous to animal PCD regulators. Among these is the plant Bax Inhibitor-1 (BI-1) protein, which possesses, like its human counterpart, the ability to suppress the lethality induced by the proapoptotic regulator Bax [3-4]. Of interest, expression of BI-1 is rapidly upregulated in plants during wounding or pathogen challenge [4]. To elucidate the role of BI-1 in the regulation of plant PCD, we undertook localization studies using a BI-1 homologue from Brassica napus, referred to as BnBI-1, and we investigated the effect of Salicylic Acid (SA), a plant hormone principally involved in defense against pathogens [5], on its subcellular distribution.

Localization studies were conducted using stable transformation of tobacco BY-2 cells or transient expression in tobacco leaves, with the fusion protein BnBI-1GFP under control of the constitutive CaMV35S promoter. Cells transformed with untargeted GFP, or GFP targeted to the ER by the way of a KDEL signal sequence at its C-terminus (ER-GFP) were used as controls. BY-2 cells expressing GFP showed diffuse fluorescence distributed throughout the cytoplasm and in the nucleoplasm (Figure 1A-B). In contrast, the fluorescence was mainly detected within and in the vicinity of the nuclear envelope as well as in the cytoplasm in BY-2 cells expressing BnBI-1GFP, while no fluorescence was observed in the nucleoplasm (Figure 1C-F). Examination of these cells at higher magnification (Figure 1E-F) showed a fine fluorescent network into the cytoplasm, which strongly suggests an association with membranes. This subcellular distribution of the fluorescent signal fits perfectly with the one observed in BY-2 cells expressing ER-GFP (Figure 1G-H). Moreover, visualization of BnBI-1GFP in tobacco leaves following transient expression (Figure 1I-J) showed an intracellular pattern of distribution closely related to the one observed in BY-2 cells expressing BnBI-1GFP or ER-GFP. In palisade mesophyll cells, BnBI-1GFP did not co-localize with the red autofluorescence from chloroplasts (Figure 1J). Moreover, use of MitoTracker to visualize mitochondria in living BY-2 cells expressing BnBI-1GFP failed to show a co-localization with mitochondria (Figure 1K). Fluorescence profile of BY-2 cells expressing BnBI-1GFP and ER-GFP was markedly different after an incubation with $125 \mathrm{mM} \mathrm{SA}$, as visualized by epifluorescence microscopy (Figure 2). Both BnBI-1GFP and ER-GFP cells treated with SA showed numerous spherical green structures, empty or full respectively (Figure 2B, D), while no such structures where visible in control cells (Figure 2A, C). Such ER reorganisation has been previously reported for virus-infected tobacco cells expressing ER-GFP [6], but this has not been linked to SA production. Taken together, our observations strongly suggest an association of BnBI-1 with the ER membranes and its nuclear envelope continuity. Our data also suggest that SA could modulate the trafficking of cellular membranes, and particularly ER membranes. In addition, this work shows that cells 
expressing a GFP targeted to the ER are good tools to visualize dynamic cellular response involving a rearrangement of intracellular membranes to generate vesicles.

[1] D.L. Vaux, Proc. Natl. Acad. Sci. USA 90 (1993) 786.

[2] R.I. Pennell and C. Lamb, Plant Cell 9 (1997) 1157.

[3] M. Kawai et al., FEBS Lett. 464 (1999) 143.

[4] P. Sanchez et al., Plant J. 21 (2000) 393.

[5] P. Reymond and E.E. Farmer, Curr. Opin. Plant Biol. 1 (1998) 404.

[6] P. Dunoyer et al., J. Virol. 76 (2002) 865.

[7] This work was supported by grants from the National Sciences and Engineering Research Council of Canada.
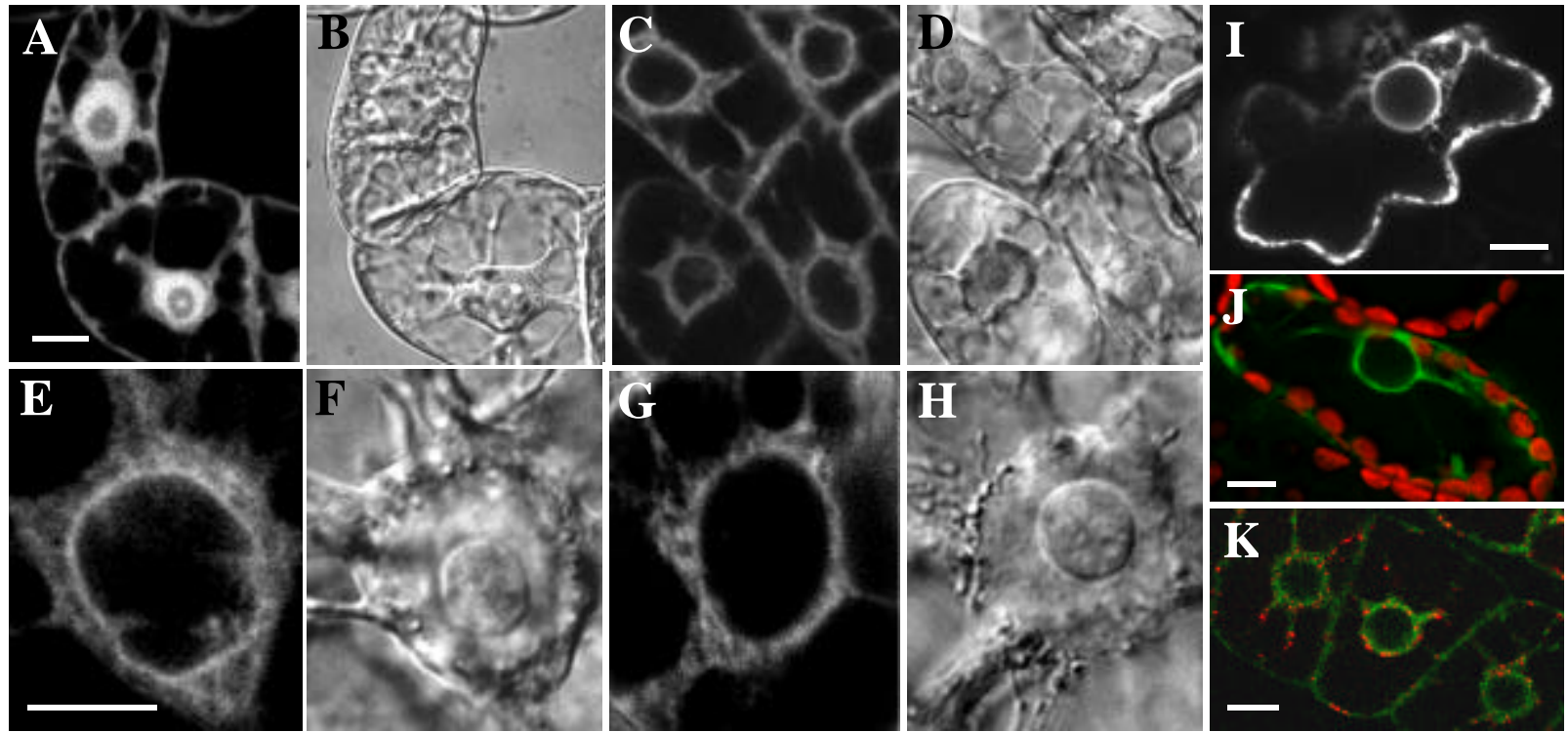

Figure 1. Localization microscopy of BnBI-1GFP in plant. Cells were viewed under a confocal microscope at an excitation wavelength of $488 \mathrm{~nm}$ (A, C, E, G, I-K) and $543 \mathrm{~nm}(\mathrm{~J}-\mathrm{K})$, or using Nomarski (B, D, F and H). A-H. Tobacco BY-2 cells stable transformed with GFP (A-B), BnBI1GFP (C-F) or ER-GFP (G-H). I-J. Transient expression of BnBI-1GFP in epidermal (I) and palisade mesophyll (J) leaf cells. In (J), fluorescence from chlorophyll and GFP are shown in red and green respectively. K. Staining of mitochondria with MitoTracker Orange (red fluorescence) in BY2 cells expressing BnBI-1GFP (green fluorescence). Bar $=10 \mu \mathrm{m}$.

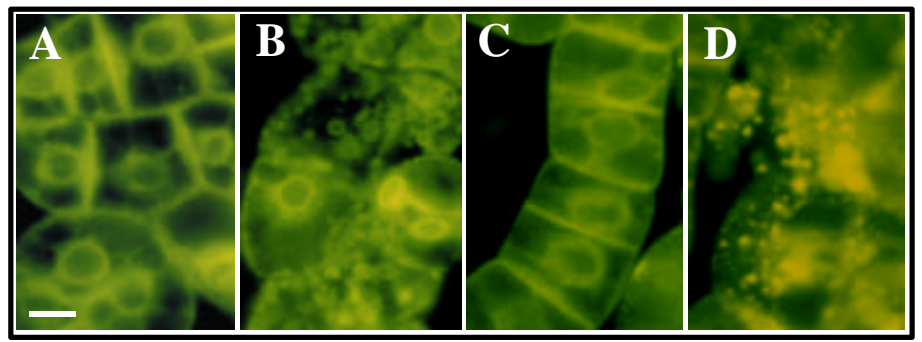

Figure 2. Salicylic acid treatment of tobacco BY-2 cells stable transformed with BnBI-1GFP (A-B) or ER-GFP (C-D). Salicylic acid was added to a final concentration of $125 \mu \mathrm{M}$ (B and D), while controls were treated with $\mathrm{H}_{2} \mathrm{O}$ (A and C). Images have been taken $9 \mathrm{hrs}$ later with a microscope equipped for epifluorescence at an excitation wavelength of $488 \mathrm{~nm}$. Bar $=10 \mu \mathrm{m}$. 